\title{
Geociências
}

\section{Caracterização microestrutural de um provável fragmento do meteorito Itutinga}

\author{
Microstructural characterization of a probable Itutinga \\ meteorite fragment
}

\section{Gilson Antônio Nunes \\ Mestre, Museu de Ciência e Técnica da Escola de Minas da Universidade \\ Federal de Ouro Preto \\ E-mail:gilson@ufop.br}

\section{Adilson Rodrigues da Costa \\ Doutor, Departamento de Engenharia Metalúrgica e de Materiais da Escola de Minas da Universidade Federal de Ouro Preto, Rede Temática de Engenharia de Materiais REDEMAT - UFOP/CETEC/UEMG E-mail: adilson@em.ufop.br}

\section{Flávio Sandro Lays Cassino}

Doutor, Departamento de Engenharia Metalúrgica e de Materiais da Escola de Minas da Universidade Federal de Ouro Preto, Rede Temática de Engenharia de Materiais REDEMAT - UFOP/CETEC/UEMG E-mail:fcassino@em.ufop.br

\section{Paulo Antônio de Souza Jr.}

Doutor, Tasmanian ICT Centre, CSIRO, Rede Temática de Engenharia de Materiais REDEMAT - UFOP/CETEC/UEMG

E-mail: paulo.desouza@csiro.au

\section{Resumo}

O Museu de Ciência e Técnica da Escola de Minas da Universidade Federal de Ouro Preto possui um fragmento de meteorito metálico com 110,76g denominado Itumirim, encontrado próximo a Itutinga, cidade do interior de Minas Gerais, distante 228km de Belo Horizonte. Realizando-se análises ao microscópio eletrônico de varredura (MEV), determinou-se a classificação estrutural do fragmento Itumirim como octaedrito médio, Om. As fases mineralógicas presentes foram determinadas recorrendo-se às análises com o detector de energia dispersiva de raios X (EDS) acoplado ao MEV e com o espectrômetro Mössbauer miniaturizado, MIMOS II. Esses resultados somados à correlação existente entre classificação estrutural e química permitiram classificar o fragmento Itumirim como pertencente ao grupo IIIAB, a mesma classificação do meteorito Itutinga.

Palavras-chave: Meteorito, meteorito metálico, Itutinga, Itumirim, MEV-EDS, Mössbauer.

\section{Abstract}

The School of Mines Museum of Science and Technology of the Federal University of Ouro Preto has, in its collection a fragment of an iron meteorite weighing $110.76 \mathrm{~g}$ named Itumirim. It was found near Itutinga, a village distant $228 \mathrm{~km}$ from Belo Horizonte in the state of Minas Gerais. SEM analysis, coupled with EDS (Energy Dispersive Spectrometry) showed that the structural class of the studied Itumirim fragment is medium octahedrite, Om. The mineralogical phases were determined using EDS and Mössbauer Spectroscopy (Miniaturized Mössbauer Spectrometer - MIMOS II). Theses studies provided information for classifying the meteorite as belonging to the IIIAB group, the same classification as the Itutinga meteorite.

Keywords: Meteorite, Iron meteorite, Itutinga, Itumirim, SEM-EDS, Mössbauer spectroscopy. 


\section{Introdução}

\subsection{Generalidades sobre os meteoritos}

Os meteoritos são fragmentos de corpos sólidos naturais (como asteróides, Lua, planetas rochosos, cometas), que, vindos do espaço, penetram a atmosfera terrestre, tornando-se incandescentes pelo atrito com os gases que compõem a atmosfera e atingem a superfície terrestre (Vieira, 2001). Quando o objeto, a rocha, é recuperado ele é denominado de meteorito. Quando se refere apenas ao objeto luminoso ele recebe o nome de meteoro. Popularmente o fenômeno luminoso é chamado de estrela cadente. Quando o objeto ainda encontra-se no espaço, em órbita do Sol ou mesmo da Terra, ele é designado, genericamente, de meteoróide.

Os meteoritos são classificados, quanto à composição química, em: rochosos ou aerólitos, quando formados basicamente de material rochoso; metálicos, também chamados de sideritos, quando formados, basicamente, da liga metálica ferro-níquel; metálicos-rochosos, ou siderólitos, quando são compostos pelas fases metálica e rochosa.

Os meteoritos metálicos ou sideritos apresentam estruturas que indicam sua formação em ambientes de elevada pressão. De fato, a formação desses meteoritos remonta a processos ocorridos logo após o período de acreção planetária. Nesse período, o corpo é formado pela aglutinação de côndrulos, materiais metálicos, partículas sólidas diversas, além de gases e materiais voláteis, e atinge considerável dimensão (Cordani \& Sioglo, 1998).

A classificação química dos meteoritos metálicos está baseada no percentual de gálio, germânio e níquel, neles presente, isto conforme Buchwald (1975). A designação desses grupos é feita por um algarismo romano seguido de uma letra do alfabeto latino. Atualmente, existem treze grupos de meteoritos metálicos relacionados à classificação química: IAB, IC, IIAB, IIC, IID, IIE, IIF, IIIAB, IIICD, IIIE, IIIF, IVA e IVB (Maran, 1991).
Nos meteoritos metálicos, algumas fases mineralógicas são comuns como a fase de minerais, destacando-se a kamacita e a taenita que são constituídas por ferro e níquel na fase a (cela unitária cúbica de corpo centrado - CCC) e b (cela unitária cúbica de face centrada CFC). Essas fases são evidenciadas em superfícies polidas de meteoritos metálicos que exibem o conhecido padrão de Widmanstätten (Buchwald, 1975).

Entretanto outros minerais também constituem os meteoritos metálicos como a troilita (FeS), cuja ocorrência se verifica normalmente em nódulos, a schreibersita [(Fe, Ni) $\left.{ }_{3} \mathrm{P}\right]$, destacando-se a cohenita $\left[(\mathrm{Fe}, \mathrm{Ni})_{3} \mathrm{C}\right]$ e a grafita $(\mathrm{C})$.

Quanto à estrutura, os meteoritos metálicos são classificados em função da largura média das lamelas de kamacita, reveladas nas superfícies desses meteoritos, lamelas preparadas metalograficamente e atacadas com solução de álcool etílico e ácido nítrico, solução conhecida por Nital.

Revelada pelo ataque químico, a estrutura de Widmanstätten é resultante, provavelmente, da fusão e resfriamento gradual dos meteoritos metálicos (Runcorn, 1978). Esse lento resfriamento, de acordo com Woolfson (1978), se dá a taxas de cerca de $1 \mathrm{~K}$ a cada milhão de anos.

\subsection{Histórico do fragmento do meteorito analisado}

O fragmento do meteorito, identificado no Museu de Ciência e Técnica da Escola de Minas da UFOP como Itumirim, não é mencionado no mais completo catálogo de meteoritos (Grady, 2000) e nem no mais recente artigo que relaciona a coleção de meteoritos brasileiros por Zucolotto (2004). Pela ficha catalográfica, esse meteorito foi encontrado a $2 \mathrm{~km}$ a sudoeste de Itutinga e doado ao museu pelo Dr. José Carlos Ferreira Gomes, que realizou a doação em 04/07/1947. A cidade Itumirim é vizinha de Itutinga, seu antigo distrito. Na mesma ocasião, foi doado ao museu pelo Dr. José Carlos o meteorito metálico Itutinga. Mesmo não estando relacionado nas publicações de referência e muito menos classificado, sabe-se que o fragmento denominado Itumirim é uma amostra de meteorito do tipo metálico pela textura externa e, principalmente, pela face metálica que exibe (resultante de algum corte), face típica dos meteoritos metálicos. Dada a proximidade do local de queda dos dois meteritos metálicos, bem como a data de doação, deve ser considerada a possibilidade de que o meteorito Itumirim seja um fragmento do meteorito Itutinga.

\section{Materiais e métodos}

Análises experimentais compatíveis foram utilizadas para alcançar o objetivo de caracterizar, microestruturalmente, o provável fragmento do meteorito Itutinga, denominado Itumirim. Considerando a natureza especial dos materiais analisados, os quais são fragmentos de rocha remanescentes da formação do Sistema Solar, com cerca de 4,57 bilhões de anos (Kirsten, 1978), sendo de origem extraterrestre, provavelmente entre as órbitas dos planetas Marte e Júpiter (Cordani \& Sioglo, 1998), a quase 500 milhões de quilômetros da Terra (Oliveira Filho, 2004), optou-se pela utilização de técnicas de análise não-destrutivas.

O meteorito em questão pertence à coleção de um museu com um dos maiores e mais expressivos acervos científicos do país, ou seja, não é apenas uma amostra, mas um objeto museológico, sobre o qual se aplicam conceitos de preservação que salvaguardam acervos dessa natureza. O fragmento analisado com apenas $110,76 \mathrm{~g}$ poderá ser identificado como um novo meteorito, fato que determina a adoção de procedimentos para garantir a preservação dessa diminuta massa. Todas essas considerações apontam, quando possível, para a utilização de técnicas de análise não-destrutivas na caracterização desses meteoritos. De fato, nenhum corte foi promovido no fragmento de meteorito analisado, garantindo sua preservação. Pode-se considerar, também, que as técnicas de preparação de amostras para análises metalográficas, como lixamento, polimento e ataque químico com 
solução de álcool etílico e ácido nítrico a $4 \%$ de ácido em volume (conhecido por Nital), a que o meteorito foi submetido, não acarretam significativo prejuízo à preservação da amostra, uma vez que incidiram apenas na superfície e em regiões diminutas.

Para o fragmento analisado, aproveitou-se a existência da superfície resultante de cortes realizados para a preparação das amostras metalográficas, dispensando a realização de outros cortes, contribuindo, novamente, para a preservação.

Na caracterização desse meteorito, procedeu-se à determinação das propriedades físicas macroscópicas, à classificação mineralógica e, finalmente, à caracterização microestrutural.

\section{Resultados e discussão}

Além da massa e das dimensões do meteorito (Tabela 1) foi determinada sua densidade, obtendo-se o valor de $6,93+0,5 \mathrm{~g} / \mathrm{cm}^{3}$. O valor obtido está razoavelmente próximo do intervalo de variação de densidade para os meteoritos metálicos, qual seja, 7,7 e 7,9g/ $\mathrm{cm}^{3}$ (Sears, 1978).

Uma possibilidade para explicar a diferença do valor encontrado para a densidade com os valores da literatura pode ser o diferenciado grau de oxidação do fragmento, bem como a presença de trincas, inclusões e vazios.

A caracterização mineralógica do meteorito foi realizada utilizando o espectrômetro Mössbauer miniaturiza- do, o MIMOS II, instalado no centro de pesquisas da mineradora Vale em Vitória - ES. Os espectros Mössbauer foram obtidos à temperatura ambiente, em geometria de retroespalhamento com uma fonte de ${ }^{57} \mathrm{Co} / \mathrm{Rh}$ de $100 \mathrm{mCi}$ de atividade. Para a análise dos espectros Mössbauer, utilizou-se o programa de ajuste de mínimos quadrados denominado NORMOS (De Souza, 2004), empregando-se um conjunto de sítios lorenztianos. Esse modelo de espectrômetro Mössbauer está sendo empregado na caracterização da superfície de Marte (Klingelhoefer, 2003), incluindo meteoritos (Schroeder, 2008).

A análise de uma série de parâmetros (Tabela 2) garante a correta interpretação dos espectros e a identificação das fases existentes, como, por exemplo, o banco de dados do Sistema de Informação Mössbauer ou Mössbauer Information System (MIS) do Centro de Dados de Efeito Mössbauer da Universidade da Carolina do Norte em Asheville, Estados Unidos. O MIS coleciona dados desde a descoberta do efeito Mössbauer, possuindo mais de 40 mil referências bibliográficas correlacionadas, sendo atualizado constantemente (Stevens et al., 1982).

Além da rápida e eficiente caracterização dessa amostra meteorítica, que contém, sabidamente, elevado percentu- al de ferro (Kracher, 1980), o MIMOS II oferece uma singular característica de operação, que permite a obtenção de dados e conseqüente geração do espectro apenas pelo contato do aparelho com a superfície a ser analisada, permitindo a realização de análises não-destrutivas, preservando, assim, a rara amostra do meteorito (Figura 1).

As análises do espectro Mössbauer da face polida desse provável fragmento do meteorito Itutinga (Figura 2) revelaram a presença de duas fases de kamacita $(\alpha-\mathrm{FeNi})$ e uma fase de taenita $(\gamma-\mathrm{FeNi})$.

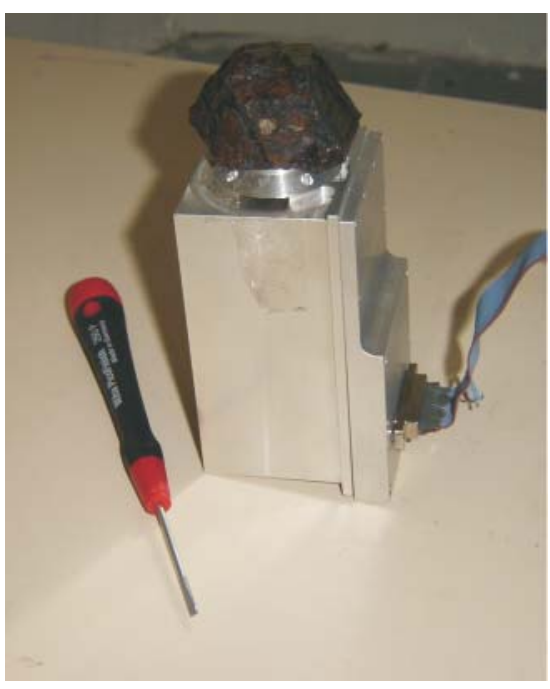

Figura 1 - Fragmento de meteorito em análise no MIMOS II, cujas dimensões são $(9 \times 5 \times 5) \mathrm{cm}$

Tabela 1 - Dimensões do fragmento de meteorito.

\begin{tabular}{c|c|c|c}
\hline Massa (g) & $\begin{array}{c}\mathbf{X} \text { - Comprimento } \\
(\mathbf{c m})\end{array}$ & $\begin{array}{c}\mathrm{Y}-\text { Largura } \\
\mathbf{( \mathbf { c m } )}\end{array}$ & $\begin{array}{c}\mathbf{Z}-\text { Altura } \\
(\mathbf{c m})\end{array}$ \\
\hline 110,76 & 4,6 & 2,6 & 2,5 \\
\hline
\end{tabular}

Tabela 2 - Parâmetros Mössbauer da análise do fragmento de meteorito.

\begin{tabular}{c|c|c|c|c|c}
\hline Fase & $\delta(\mathbf{m m} / \mathbf{s}) \mathbf{+ 0 , 0 2}$ & $\Delta(\mathbf{m m} / \mathbf{s}) \mathbf{\pm 0 , 0 2}$ & $\Gamma(\mathbf{m m} / \mathbf{s}) \pm \mathbf{0 , 0 2}$ & $\mathbf{B}_{\mathrm{hf}}(\mathbf{m m} / \mathbf{s}) \pm \mathbf{0 , 3}$ & Área (\%) \\
\hline$\alpha-\mathrm{FeNi}$ & $-0,05$ & 0,04 & 0,4 & 33,7 & 69 \\
\hline$\alpha-\mathrm{FeNi}$ & $-0,04$ & 0 & 0,4 & 0 & 9 \\
\hline$\gamma$-FeNi & $-0,07$ & $-0,16$ & 0,31 & 33,8 & 22 \\
\hline
\end{tabular}

Onde: $\delta$ - deslocamento isomérico, $\Delta$ - desdobramento quadrupolar, $\Gamma$ - largura de linha na altura média, $B_{h f}$ - campo magnético hiperfino, Área - área do subespectro. 
Utilizou-se, para determinar outras fases mineralógicas existentes no meteorito, o microscópio eletrônico de varredura acoplado ao detector de energia dispersiva de raios X (MEV/EDS). Com esses resultados, identificaram-se fases minerais que não podem ser detectadas pela espectrometria Mössbauer. Além disso, o EDS acoplado ao MEV possibilita realizar a análise química semiquantitativa com conhecimento da posição em que os dados são obtidos.

Após a preparação metalográfica do fragmento de meteorito, o mesmo foi levado ao MEV/EDS do Laboratório de Microanálises do Departamento de Engenharia Geológica da Escola de Minas da UFOP. A partir das imagens geradas pelo microscópio, identificouse, no fragmento em questão, uma fase semelhante à kamacita, obtida por quatro medidas nessa lamela (Figura 3).

Os resultados das contagens para o fragmento de meteorito, além de confirmarem a presença da kamacita, indicaram, ainda, a presença de alumínio, provavelmente resíduos do abrasivo utilizado na operação de polimento da amostra. A Figura 4 apresenta os espectros EDS para os pontos de 1 a 4 do provável fragmento do meteorito Itutinga.

No framento de meteorito, identificou-se, ainda, a existência da fase plessita (Figura 5). O espectro obtido por MEV/EDS, nessa fase, ponto 2, encontra-se em destaque na Figura 6. A presença recorrente de alumínio, no fragmento de meteorito, agora na plessita, reforça a possibilidade de contaminação por resíduos do abrasivo, no caso óxido de alumínio $\left(\mathrm{Al}_{2} \mathrm{O}_{3}\right)$

Valendo-se das análises do fragmento no MEV/EDS, para caracterização química, efetuou-se, também, a caracterização microestrutural desse meteorito (Figura 7).

As medidas lineares da largura média das lamelas de kamacita desse meteorito com cerca de 1,0mm são compatíveis com as lamelas do meteorito Itutinga (Grady, 2000) e com o intervalo correspondente à classe octaedrito mé-

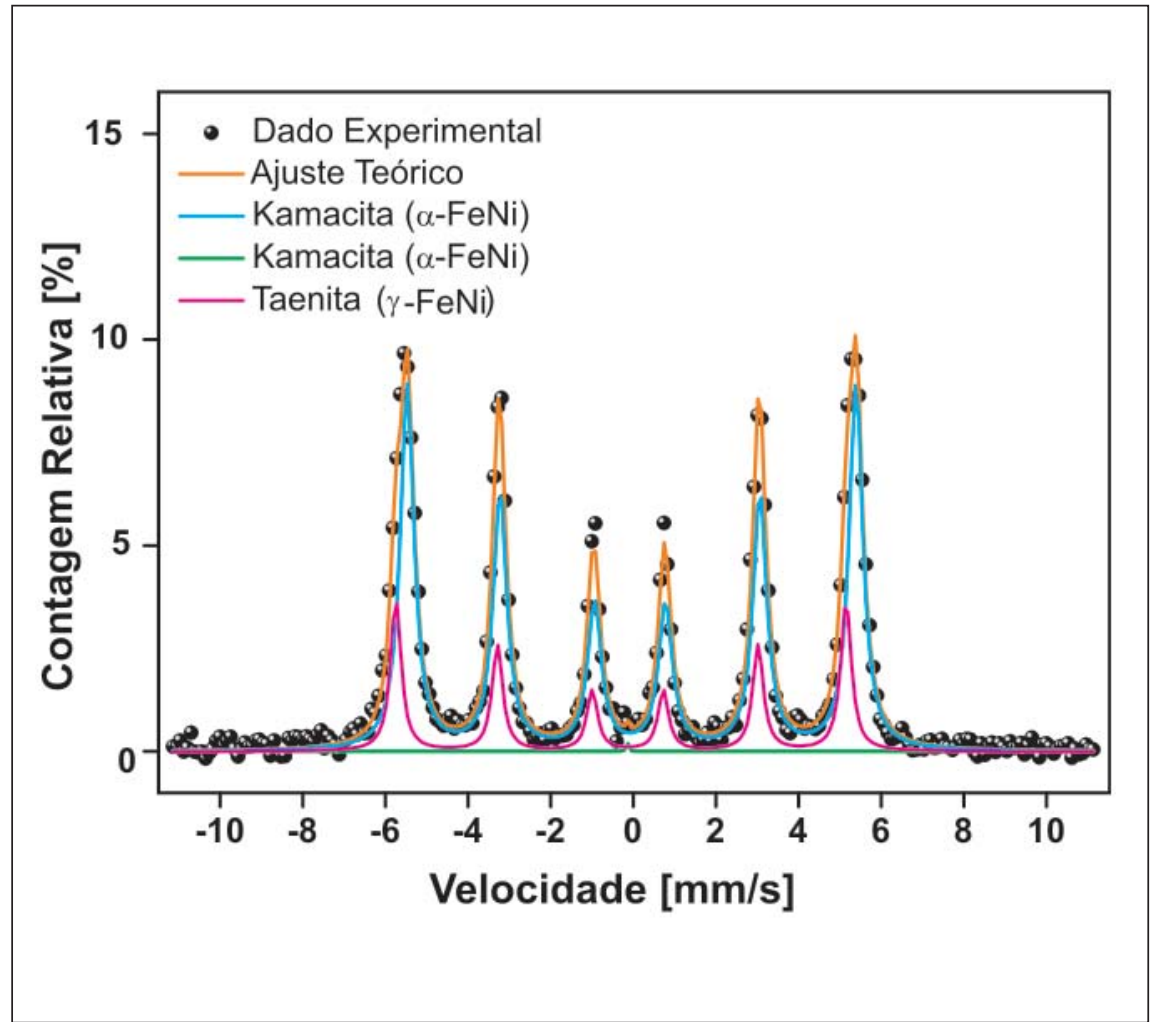

Figura 2 - Espectro Mössbauer da face polida do fragmento de meteorito.

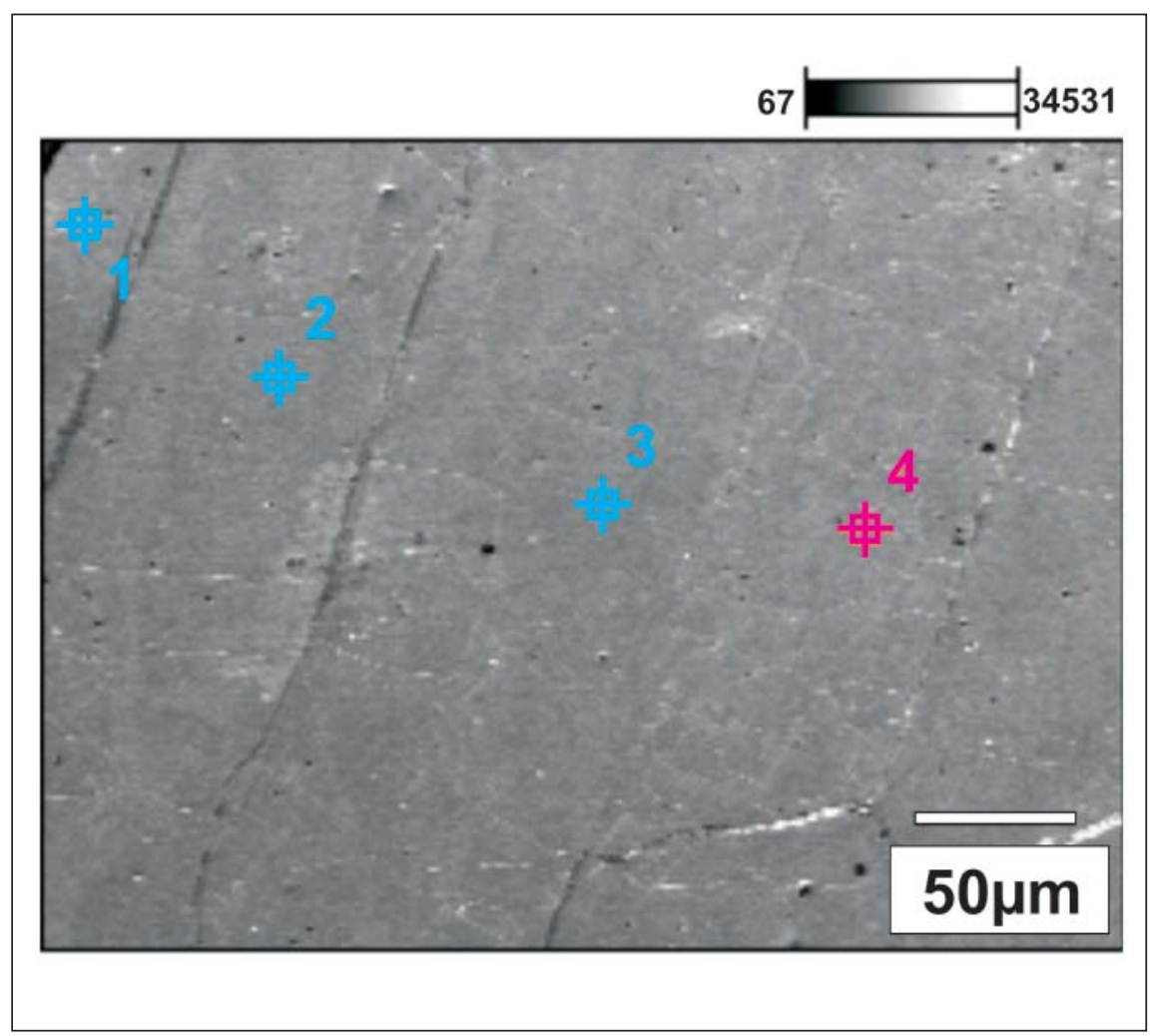

Figura 3 - Imagem e pontos de contagem do MEV/EDS para o fragmento de meteorito. 
Gilson Antônio Nunes et al.

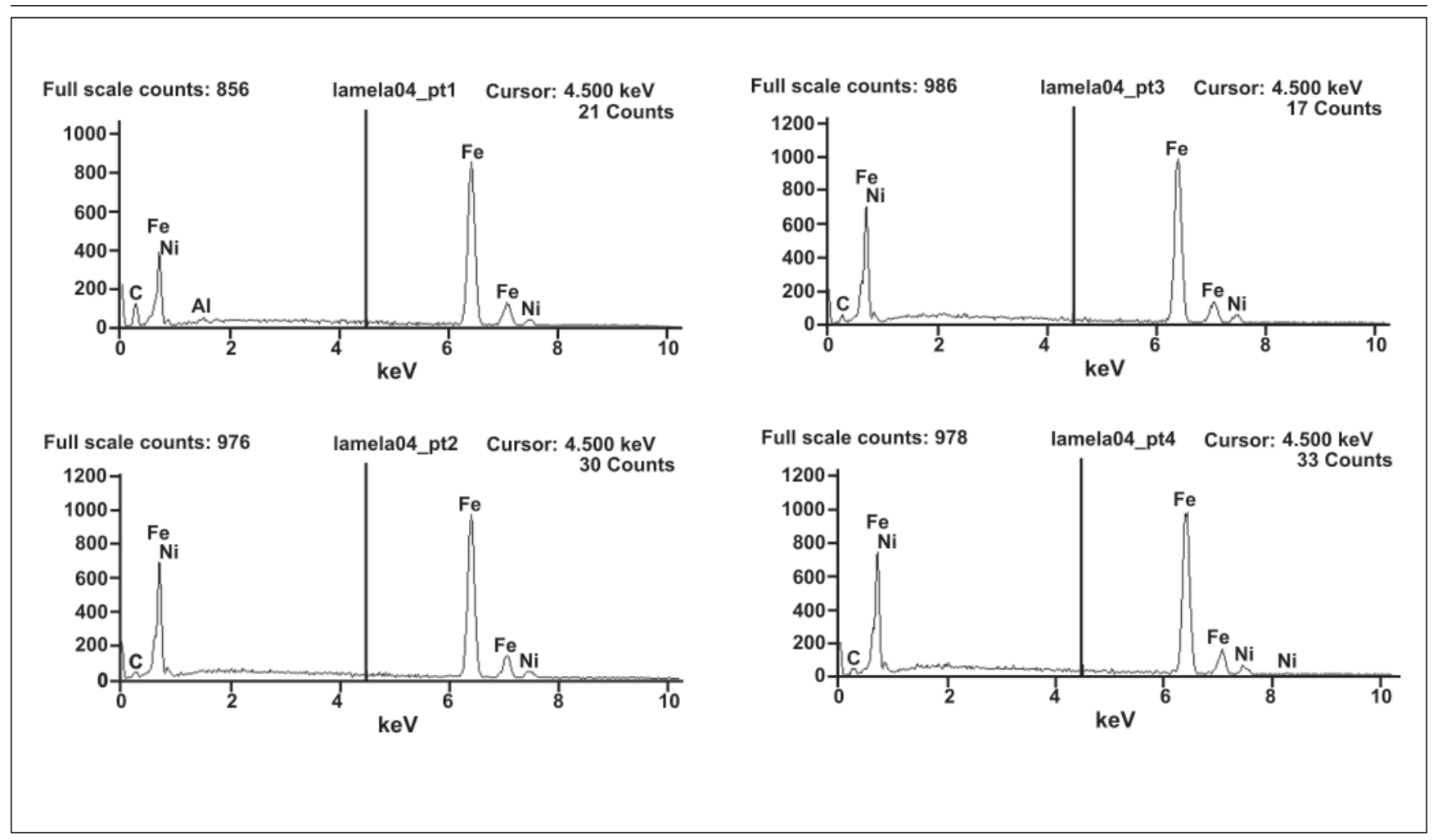

Figura 4 - Espectros para os pontos 1 a 4 do fragmento de meteorito gerados por MEV/EDS.

dio (0,5 a 1,3mm) relativa à classificação estrutural. Tais medidas foram obtidas em dois planos e com correções de ângulos, uma vez que se trata de lamelas em uma estrutura octaédrica.

Em função da correspondência existente entre a classificação estrutural e química, os meteoritos metálicos da classe octaedrito médio são do grupo IIIAB quanto à classificação química (Sears, 1978).

Portanto o fragmento de meteorito metálico analisado pode ser classificado como pertencente à classe octaedrito médio, Om, e grupo IIIAB. A relação L/W (comprimento da lamela dividido por sua largura) para o fragmento é de, aproximadamente, 10 .

\section{Conclusão}

Realizadas a revisão bibliográfica e as diversas análises, concluiu-se que o fragmento de meteorito analisado é um meteorito metálico da classe octaedrito médio, Om, e do grupo IIIAB, a mes-

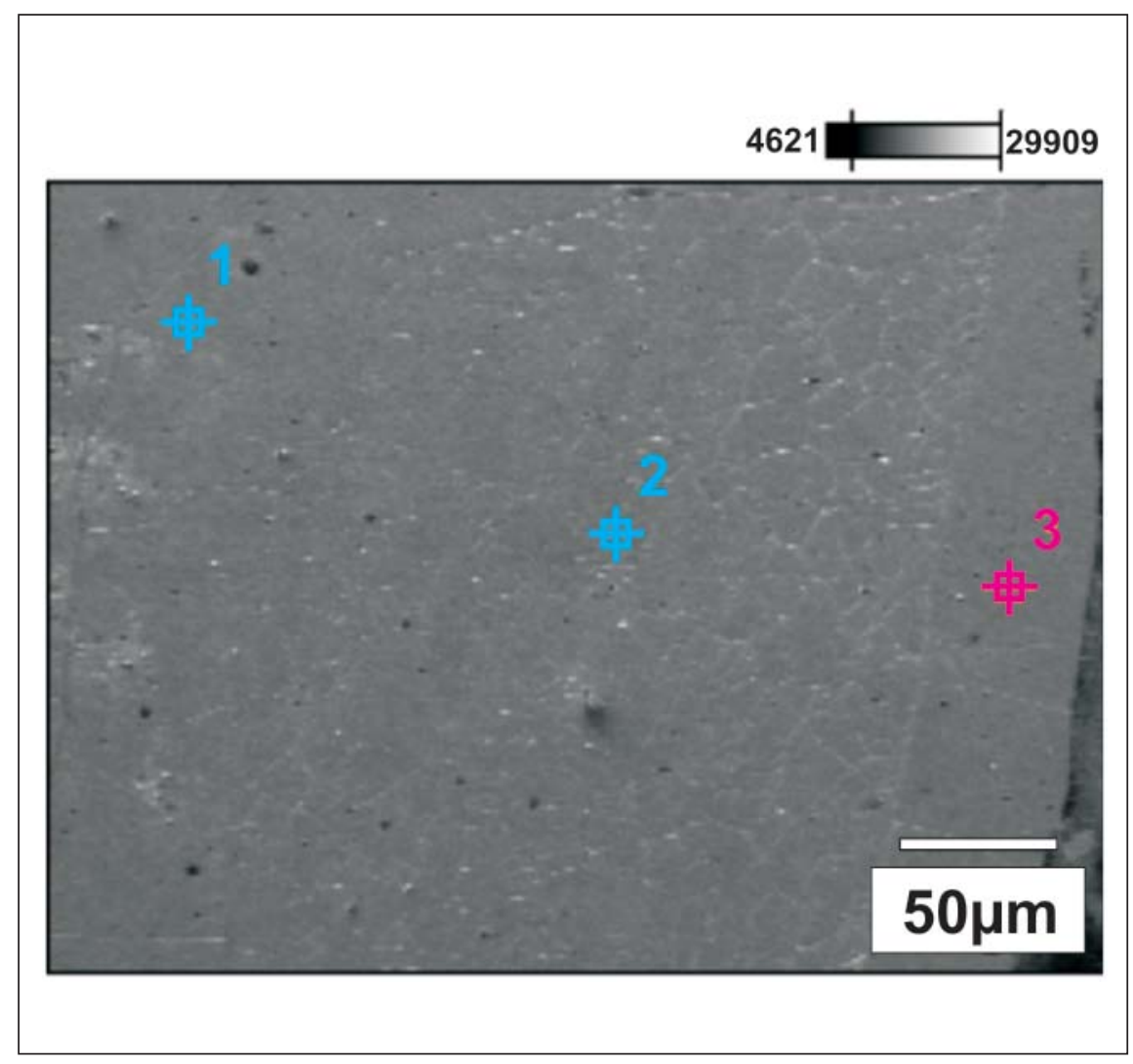

Figura 5 - Imagem de plessita no fragmento de meteorito. 


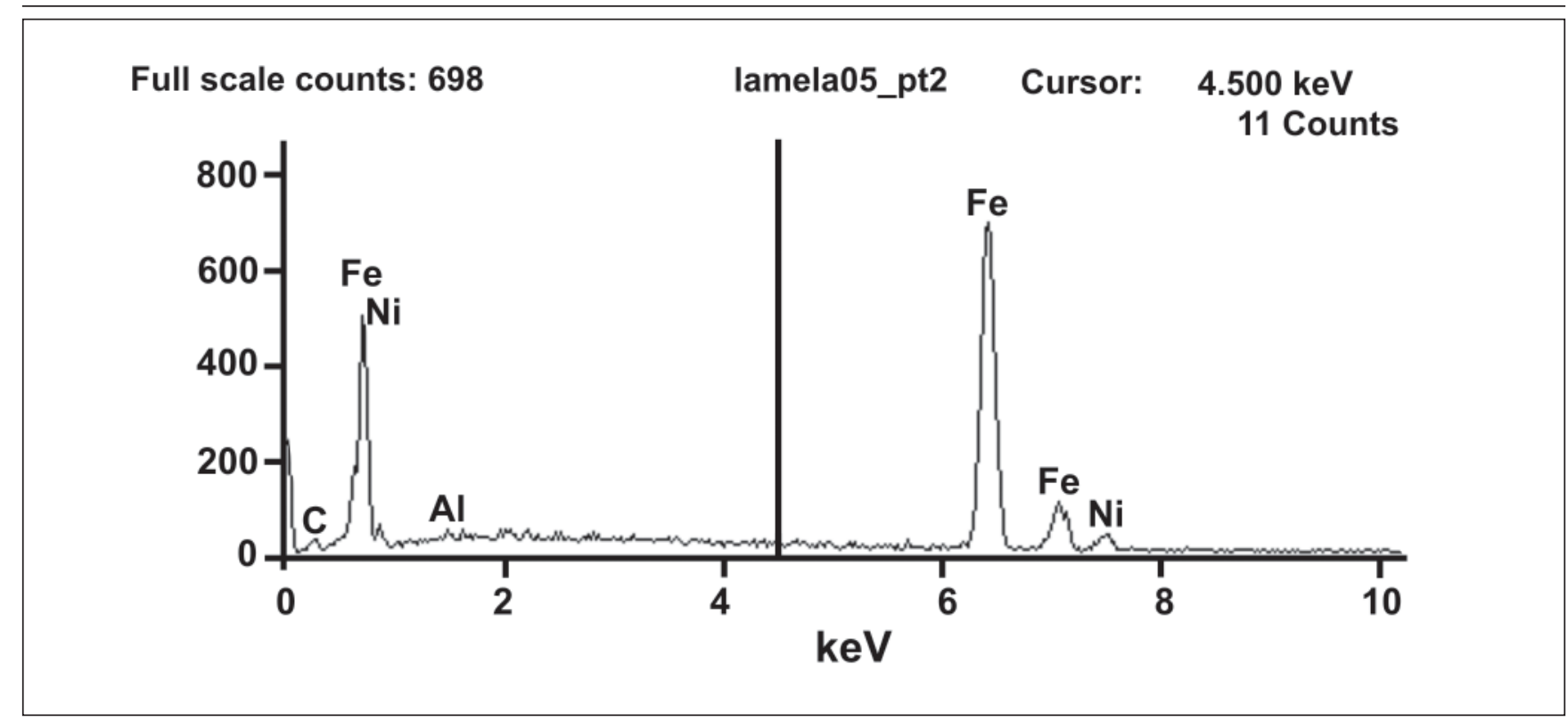

Figura 6 - Espectro da fase plessita localizada no fragmento de meteorito.

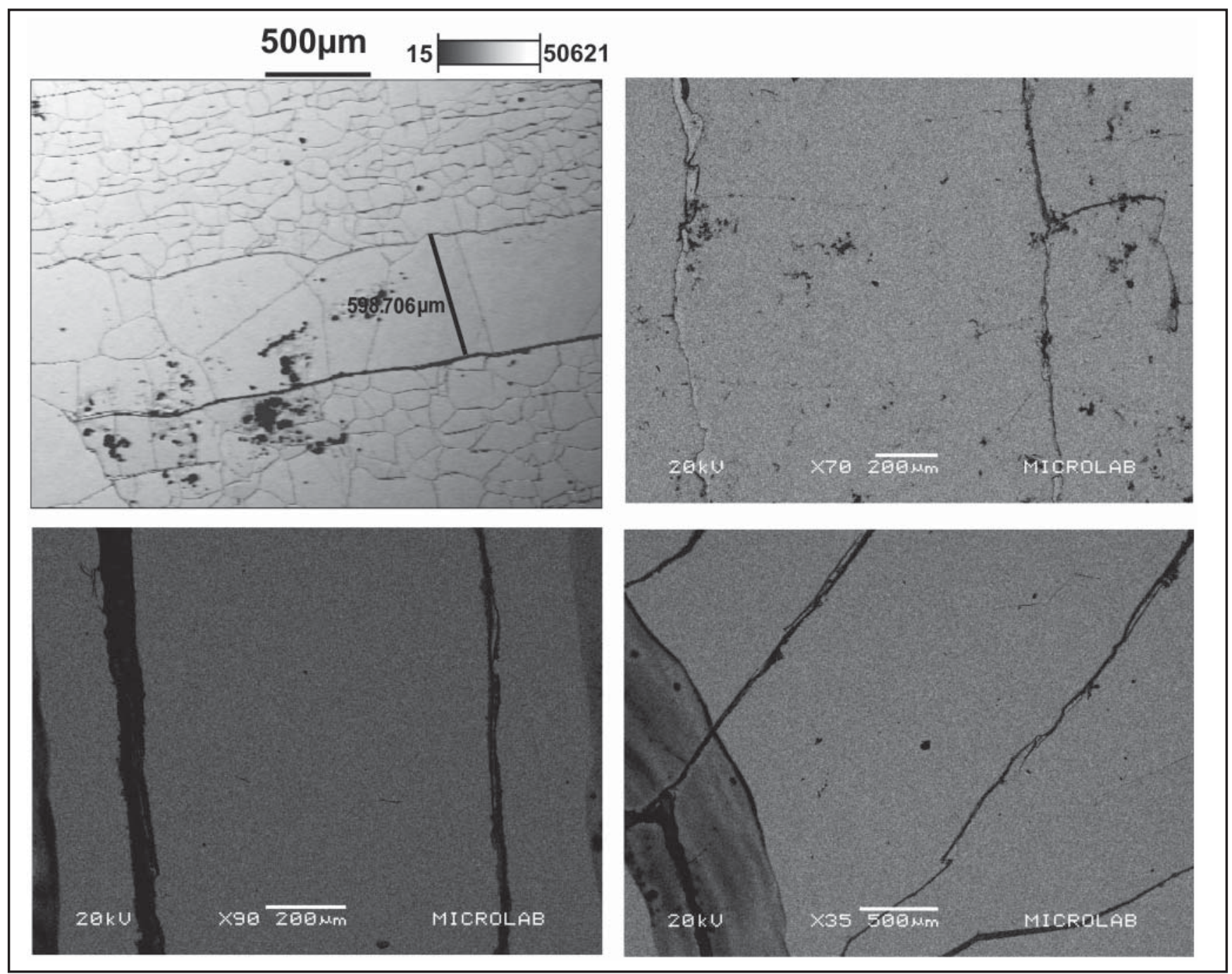

Figura 7 - Medida de lamelas de kamacita do meteorito.

430 REM: R. Esc. Minas, Ouro Preto, 63(3): 425-431, jul. set. 2010 
ma classificação do meteorito Itutinga (Buchwald, 1975). Apesar de não ser possível afirmar taxativamente, é provável que o meteorito Itumirim seja um fragmento do meteorito Itutinga.

\section{Agradecimentos}

Ao Laboratório de Tratamento Térmico do Departamento de Engenharia Metalúrgica e de Materiais, ao Laboratório de Microanálises do Departamento de Engenharia Geológica e ao Museu de Ciência e Técnica da Escola de Minas da UFOP. Agradecemos, ainda, ao Centro de Pesquisas da Vale e ao Museu Nacional da Universidade Federal do Rio de Janeiro.

\section{Referências bibliográficas}

BUCHWALD, V. F. Handbook of iron meteorites, their history, distribuition, composition, and structure. Berkeley: University of California Press, 1975. 3v., 1418p.

CORDANI, U. G., SIOGLO, J. B. Composição e estrutura interna de Marte. In: Marte Novas Descobertas. São Paulo: Diagrama \&Texto, Instituto Astronômico e Geofísico da Universidade de São Paulo, 1998. 243p.
DE SOUZA, Jr. P. A. Extraterrestrial and Terrestrial Outdoor Applications of Mössbauer Spectroscopy. Doktors der Naturwissenschaften, Johannes Gutenberg Universität, Mainz, 2004. 152 p.

GRADY, M. M (Ed.). Catalogue of meteorites. Cambridge: Cambridge University Press, 2000. 689 p.

KLINGELHOEFER, G. et al. Athena MIMOS II Mössbauer spectrometer investigation. Journal of Geophysical Research, v. 108, n. E12, 8067, doi:10.1029/2003JE002138, 2003.

KRACHER, A., WILLIS, J., WASSON, J. T. Chemical Classification of Iron Meteorites IX. A New Group (IIF), Revision of IAB and IIICD, and Data on 57 Aditional Irons. In: Geochemistry et Cosmochemistry Acta. London: Pergamon Press Ltd., 1980. v.44, p. $773-787$.

KIRSTEN, T. Time and the solar system. In: The Origin of Solar System. Chichester: John Wiley and Sons Ltd., 1978. p. 267-346.

MARAN, S.P. (Editor). The astronomy and astrophysics encyclopedia. Cambridge: Cambridge University Press, 1991. 1002p.

OLIVEIRA FILHO, K. S., SARAIVA, M. F. O. Astronomia e astrofísica. São Paulo: Editora Livraria da Física, 2004. 557 p.

RUNCORN, S. K. Evidence from the Early Moon. In: The Origin of Solar System. Chichester: John Wiley and Sons Ltd., 1978. p. 459.

SCHROEDER, Ch. et al. Meteorites on Mars observed with the Mars Exploration. Rovers. Journal of Geophysical Research - Planets, Vol. 113, E06S22, doi:10.1029/2007JE002990, 2008.

SEARS, D. W. The nature and origin of meteorites. Bristol: J. W. Arrowsmith Ltd., 1978. $187 \mathrm{p}$.

STEVENS, J. G., POLLAK, H., ZHE, L., STEVENS, V. E., WHITE, R. M., GIBSON, J. L. Mineral data. Asheville: Mössbauer Effect Data Center of University of North Carolina, 1982.

VIEIRA, C. J. Meteoritos Brasileiros. In: ENCONTRO NACIONAL DE ASTRONOMIA, 4. Anais... Salvador: Associação de Astrônomos Amadores da Bahia - AAAB, p. 29 a 38, 2001.

WOOLFSON, M. M. Evolution of the solar system. In: The Origin of Solar System. Chichester: John Wiley and Sons Ltd., 1978. p. 612.

ZUCOLOTTO, M. E. Brazilian Meteorites. In: Meteoritics and Planetary Science. Tucson: Planetary Society, v. 39, p. 119A, 2004.

Artigo recebido em 06/03/2009 e aprovado em 19/03/2010.

\section{A REM tem novo endereço: FUNDAÇÃO GORCEIX - REM} Rua Carlos Walter Marinho Campos, 57 Bairro: Vila Itacolomy 35400-000 - Ouro Preto - MG $\begin{array}{lll}\text { (31) } 3551-4730 & \text { (31) 3559-7408 }\end{array}$ www.rem.com.br 